\title{
INDICE DELL'ANNATA
}

\begin{tabular}{|c|c|c|}
\hline & $n$. & pp. \\
\hline $\begin{array}{l}\text { Adornato Francesco, Intervento pubblico, distretti Ogm free } e \\
\text { accordi negoziali }\end{array}$ & $1 / 2$ & $19-32$ \\
\hline Adornato Francesco, Cultura alimentare e politiche agricole & 3 & $5-10$ \\
\hline $\begin{array}{l}\text { Altiero Salvatore, Sulla recente normativa cinese in materia di sicu- } \\
\text { rezza alimentare }\end{array}$ & $1 / 2$ & 235-279 \\
\hline $\begin{array}{l}\text { Arabia Aida Giulia, Desideri Carlo, Le materie dello "sviluppo } \\
\text { economico" alla prova del federalismo fiscale }\end{array}$ & $1 / 2$ & 115-131 \\
\hline $\begin{array}{l}\text { Altili Priscilla, Il ruolo dei Paesi in via di sviluppo per la protezione } \\
\text { delle risorse biogenetiche e dei diritti delle comunità locali che } \\
\text { le hanno preservate }\end{array}$ & $1 / 2$ & 185-206 \\
\hline $\begin{array}{l}\text { Borghi Paolo, Gli Ogm, le nyuove congiunzioni astrali e il fuoco } \\
\text { sotto la cenere }\end{array}$ & $1 / 2$ & $7-16$ \\
\hline Bruno Francesco, Le etichette degli alimenti Ogm & $1 / 2$ & $47-53$ \\
\hline $\begin{array}{l}\text { Carbone Anna, La multifunzionalità dell'agricoltura europea: } \\
\text { riflessioni sul contributo del settore primario alla costruzione } \\
\text { dell'identità culturale }\end{array}$ & $1 / 2$ & $133-144$ \\
\hline $\begin{array}{l}\text { Ceccarelli Salvatore, Biodiversità, miglioramento genetico partecipa- } \\
\text { tivo e diritto al cibo. (Chi decide cosa mangerai a cena?) }\end{array}$ & 3 & $77-90$ \\
\hline $\begin{array}{l}\text { Corleto Francesco, I contratti derivati come strumenti di gestione del } \\
\text { rischio nei mercati agricoli. (Possibili applicazioni nelle borse } \\
\text { merci italiane) }\end{array}$ & $1 / 2$ & $159-184$ \\
\hline $\begin{array}{l}\text { de Beaumont Maria Ludovica, La disciplina giuridica dei fondi rustici } \\
\text { in Cina }\end{array}$ & 3 & $141-163$ \\
\hline $\begin{array}{l}\text { Fanelli Rosa Maria, Similarità e convergenza dei consumi alimentari } \\
\text { in Europa }\end{array}$ & $1 / 2$ & $145-158$ \\
\hline $\begin{array}{l}\text { Germanò Alberto, Il cibo nel diritto internazionale del mercato dei } \\
\text { prodotti agricoli: disciplina e controversie }\end{array}$ & $1 / 2$ & $85-113$ \\
\hline $\begin{array}{l}\text { Lattanzi Pamela, Distretti Ogm free: un'opportunità di differenziazione } \\
\text { del territorio }\end{array}$ & $1 / 2$ & $55-61$ \\
\hline
\end{tabular}


Matthews Glenn Jane, “Government Wrongs”: Civil liability for Gmo regulation in Canada

n. $\quad$ pp.

Nieddu Martino, De la raffinerie végétale à une bioraffinerie doublement verte?

$1 / 2 \quad 207-234$

aoloni Lorenza, Farmers' Rights, tutela della biodiversità e salvaguardia delle risorse genetiche: l'esperienza del Canada

icoveri Giovanna, Beni comuni e nuovo modello di sviluppo

Salvi Sergio, La "rivoluzione verde" di Nazareno Strampelli

91-106

Sirsi Eleonora, Quando la contaminazione da Ogm è "tecnicamente inevitabile": riflessioni in vista dell'adozione di "misure di coesistenza" nelle Regioni italiane

Superina Irene, Plant Genetic Resources for Food and Agriculture: legal and institutional frame and new perspectives

Testa Ugo, Il progetto Life Ambiente Sapid: i dubbi di una difficile coesistenza Ogm/no Ogm

Valletta Marco, La nouvelle réglementation des Ogm de l'Union européenne 